\title{
Inhibition of neointimal formation after balloon injury by cilostazol, accompanied by improvement of endothelial dysfunction and induction of hepatocyte growth factor in rat diabetes model
}

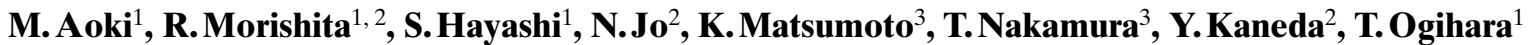 \\ ${ }^{1}$ Department of Geriatric Medicine, Graduate School of Medicine, Osaka University, Osaka, Japan \\ ${ }^{2}$ Division of Gene Therapy Science, Graduate School of Medicine, Osaka University, Osaka, Japan \\ ${ }^{3}$ Division of Biochemistry, Department of Oncology, Biomedical Research Center, Graduate School of Medicine, \\ Osaka University, Osaka, Japan
}

\section{Abstract}

Aims/hypothesis. Cilostazol, a well-known phosphodiesterase type 3 (PDE3) inhibitor for the treatment of peripheral arterial disease, has vasodilator properties and an anti-proliferative action on the growth of vascular smooth muscle cells. In this study, we tested whether cilostazol inhibits neointimal formation and improves endothelial dysfunction after balloon injury in non-diabetic and diabetic rats.

Methods. Cilostazol or vehicle was administered to non-diabetic and streptozotocin-induced diabetic rats from 7 days before to 14 days after balloon injury of the carotid artery. We focused on the expression of hepatocyte growth factor to explore how cilostazol improved endothelial dysfunction. Also, we studied the effects of cilostazol on hepatocyte growth factor production in in vitro experiments.

Results. At 14 days after injury, the ratio of neointimal to medial area was decreased in rats treated with cilostazol in non-diabetic and diabetic animals. The impaired response to acetylcholine in balloon injured vessels was improved by cilostazol in non-diabetic and dia- betic rats $(p<0.05)$. Vascular hepatocyte growth factor concentration was decreased in injured vessels of non-diabetic rats compared to uninjured vessels. Moreover, hepatocyte growth factor was further decreased in injured vessels of diabetic rats as compared to those of non-diabetic rats $(p<0.05)$. Of note, administration of cilostazol attenuated the decrease in hepatocyte growth factor concentration in injured vessels of both non-diabetic and diabetic rats $(p<0.01)$. Increase in vascular hepatocyte growth factor by cilostazol was confirmed by in vitro experiments showing that cilostazol increased hepatocyte growth factor concentration in cultured human vascular smooth muscle cells, accompanied by cAMP accumulation. Conclusion/interpretation. Our study shows that the increase in vascular hepatocyte growth factor by cilostazol could improve abnormal growth of vascular smooth muscle cells and endothelial dysfunction through rapid regeneration of endothelial cells. [Diabetologia (2001) 44: 1034-1042]

Keywords Restenosis, Remodelling, HGF, endothelial dysfunction, diabetes.
Received: 2 March 2001 and in revised form: 23 April 2001

Corresponding author: Ryuichi Morishita, M.D., Ph.D., Associate Professor, Department of Geriatric Medicine, Graduate School of Medicine, Osaka University, 2-2 Yamada-oka, Suita 565-0871, Japan, E-mail: morishit@geriat.med.osakau.ae.jp

Abbreviations: $\mathrm{PDE}_{3}$, phosphodiesterase type 3 inhibitor; HGF, hepatocyte growth factor; PTCA, Percutaneous transluminal coronary angioplasty; VEGF, vascular endothelial growth factor
Percutaneous transluminal coronary angioplasty (PTCA) greatly contributes to the therapy for ischaemic heart disease. However, restenosis or reocclusion after balloon injury remains a serious problem [1]. The major mechanisms of chronic restenosis have been reported to be prolifeation and migration of vascular smooth muscle cells (VSMC) stimulated by numerous cytokines [2-4]. Since platelets are thought to play an important part in the proliferation of VSMC by releasing these growth factors, antiplatelet drugs have often been used clinically. However, the effects of these drugs on restenosis in clinical 
fields are still controversial. Among these drugs, cilostazol, a phosphodiesterase type $3\left(\mathrm{PDE}_{3}\right)$ inhibitor, is thought to be useful for restenosis. It increases of the concentration of cyclic AMP (cAMP) by selectively blocking PDE3, resulting in the inhibition of platelet aggregation. As cilostazol is also known to suppress the growth of VSMC in vitro [5-7] and in vivo [8], some investigators have examined whether cilostazol can prevent restenosis in clinical situations. Despite the small number of patients, several groups have reported the inhibition of restenosis by cilostazol [9-15]. The molecular mechanisms by which cilostazol prevents restenosis, however, are still not known.

Endothelial function is also important for the pathogenesis of restenosis, since endothelial cells act as a biological barrier inhibiting the growth of VSMC, maintaining vascular tonus and preventing platelet adhesion $[16,17]$. Therefore, rapid regeneration or seeding of endothelial cells has potential therapeutic value for treating restenosis. Administration of recombinant vascular endothelial growth factor (VEGF) or gene transfer of VEGF resulted in the inhibition of neointimal formation, as a result of re-endothelialization [18-20]. In addition, we recently reported that gene transfer of hepatocyte growth factor (HGF) into a rat balloon injury model also resulted in the inhibition of neointimal formation and improvement of endothelial dysfunction through acceleration of endothelial regeneration [21]. Regeneration of endothelial cells should thus be considered as a target to treat restenosis. From this viewpoint, diabetes mellitus, or hyperglycaemia, is problematic, as diabetes mellitus and hyperglycaemia lead to endothelial cell death and endothelial dysfunction $[22,23]$. We focused on the effect of cilostazol on endothelial dysfunction after balloon injury in a diabetes model, as diabetic patients who have severe endothelial damage often undergo angioplasty. Thus, we studied whether cilostazol inhibits neointimal formation after balloon injury and improves endothelium-dependent vasodilation in diabetic rats the role of tissue $\mathrm{HGF}$ in injured vessels in diabetic rats, and the effect of cilostazol on the regulation of HGF in human cultured VSMC and endothelial cells.

\section{Materials and methods}

Protocol of in vivo experiments. Cilostazol $\left(100 \mathrm{mg} \cdot \mathrm{kg}^{-1}\right.$. day $^{-1}$ ) or saline as vehicle was orally administered twice a day. Balloon injury was carried out 1 week after treatment with cilostazol and administration of cilostazol or vehicle was continued until rats were killed ( 2 weeks after balloon injury). Experimental groups were divided into non-diabetic rats (SpragueDawley rats, 400-500 g; Charles River Breeding Laboratories, Wilmington, Mass., USA) without balloon injury treated with vehicle, non-diabetic rats with balloon injury treated with vehicle, non-diabetic rats with balloon injury treated with cilostazol, diabetic rats without balloon injury treated with vehi- cle, and diabetic rats with balloon injury treated with cilostazol.

Diabetes was induced by a single i.p. injection of streptozotocin $(100 \mu \mathrm{g} / \mathrm{g}$ body weight in $50 \mathrm{mmol} / \mathrm{L}$ citric acid buffer, $\mathrm{pH}$ 4.5) [24] 4 weeks before balloon injury. Then, 1 week later blood was obtained periorbitally after $8 \mathrm{~h}$ of fasting. Only rats having a blood glucose concentration above $3000 \mathrm{mg} / \mathrm{l}$ were kept in the protocol and randomized for experiments. The rats were maintained and bred under conventional conditions with food and free access to water, except on days of blood sampling and a 12-h light to 12 -h dark photoperiod. Glucose assay was done as recommended by the manufacturer (Sigma Immunochemicals, St. Louis, Mo., USA). At the end of the experiments, the blood glucose concentration of diabetic rats treated with vehicle or cilostazol was $5410+190 \mathrm{mg} / \mathrm{l}$ and $5340+170 \mathrm{mg} / \mathrm{l}$, respectively (blood glucose concentration of non-diabetic rats treated with cilostazol was $1710+6 \mathrm{mg} / \mathrm{l}$ ). The final body weight of diabetic and non-diabetic rats was $475 \pm 9 \mathrm{~g}$ and $367 \pm 9 \mathrm{~g}$, respectively $(p<0.01)$. There were no differences between body weights of rats treated by vehicle and those treated with cilostazol. The size of carotid arteries in each group was not different.

Procedure of balloon injury to carotid artery. A 2-French Fogarty catheter was used to induce vascular injury in male Sprague-Dawley rats (400-500 g; Charles River Breeding Lab.) [25, 26]. These rats were anaesthetized with ketamine and the left common carotid artery was surgically exposed. Vascular injury of the common carotid artery was produced by the passage and inflation of a balloon catheter (2-French Fogarty catheter) three times through an arteriotomy in the external carotid artery $[25,26]$. The injured segment was transiently isolated by temporary ligatures. No adverse neurological or vascular effects were observed in any animal undergoing this procedure.

At 2 weeks after injury, each carotid artery was processed for the measurement of HGF concentration and morphological study. For histological analyses, a segment of each artery was perfusion-fixed with $4 \%$ paraformaldehyde at physiological pressure $(110 \mathrm{mmHg})$ and subsequently processed. Medial and lumen area were measured with a PowerMac Lab after staining with haematoxylin. The medial area was readily demarcated as the vessel area between the internal and external elastic laminae. At least three individual sections from the middle of the arterial segments were analysed. Animals were coded so that the analysis was done without knowledge of which treatment each individual animal had received.

Animal care. This study was carried out under the supervision of the Animal Research Committee in accordance with the Guideline on Animal Experiments of Osaka University Medical School and the Japanese Government Animal Protection and Management Law (No. 105).

Measurement of HGF concentration in blood vessels. Two weeks after injury, the vessels of each group were promptly removed without excess fat after perfusion from the apex of the heart with saline, and frozen in liquid nitrogen. The tissue was thawed at $4{ }^{\circ} \mathrm{C}$, weighed, and homogenized by polytron in assay solution. Each specimen was centrifuged at $20000 \cdot \mathrm{g}$ for $30 \mathrm{~min}$ at $4{ }^{\circ} \mathrm{C}$ to remove the lysates. The concentration of HGF in the blood vessels was determined by enzyme-immunoassay using anti-rat HGF antibody [27, 28].

Evaluation of vasodilator properties in response to acetylcholine. Freshly harvested vessels were cleaned of fat and connective tissue, cut into helical strips, and mounted in $30 \mathrm{ml}$ organ baths, containing Krebs-Henseleit buffer (KNB; 120 mmol/l 
$\mathrm{NaCl}, 4.7 \mathrm{mmol} / \mathrm{l} \mathrm{KCl}, 2.5 \mathrm{mmol} / \mathrm{l} \mathrm{CaCl}_{2}, 1.2 \mathrm{mmol} / 1 \mathrm{MgSO}_{4}$, $1.2 \mathrm{mmol} / 1 \mathrm{KH}_{2} \mathrm{PO}_{4}, 25 \mathrm{mmol} / 1 \mathrm{NaHCO}_{3}, 5.5 \mathrm{mmol} / 1$ glucose, $\mathrm{pH}$ 7.4) maintained at $37{ }^{\circ} \mathrm{C}$ and oxygenated with $95 \% \mathrm{O}_{2}$, $5 \% \mathrm{CO}_{2}$ [29]. Vessels were equilibrated for $60 \mathrm{~min}$ with changes of bathing fluid every $15 \mathrm{~min}$. Isometric tension studies were done using a Grass model 7D polygraph. Optimal resting tension was determined in baseline studies and the response to vasoactive drugs was then determined. Cumulative dose-response curves to phenylephrine (PE; $10^{-9}$ to $10^{-4} \mathrm{~mol} / \mathrm{l}$ ) were established. The vessels were then submaximally precontracted with PE (typically $3 \cdot 10^{-6} \mathrm{~mol} / \mathrm{l}$ ), and endothelial function was evaluated by means of vascular relaxation to acetylcholine (Ach; $10^{-9}$ to $10^{-4} \mathrm{~mol} / \mathrm{l}$ ). Nitric oxide mediation of acetylcholine responses was confirmed by blocking Ach-induced relaxation with $\mathrm{N}$-methyl-L-arginine $(1 \mathrm{mmol} / \mathrm{l})$, a specific competitive inhibitor of nitric oxide synthase. Contractile responses were measured from the polygraph chart and expressed as a percentage of the maximal contraction, or for relaxation as a percentage of the precontracted tension.

Cell culture. Human aortic VSMC (passage 5) and human aortic endothelial cells (passage 5) were obtained from Clonetics (San Diego, Calif., USA) and cultured in modified MCDB131 medium supplemented with $5 \%$ fetal calf serum, $100 \mathrm{U} / \mathrm{ml}$ penicillin, $100 \mathrm{mg} / \mathrm{ml}$ streptomycin, $10 \mathrm{ng} / \mathrm{ml}$ epidermal growth factor, $2 \mathrm{ng} / \mathrm{ml} \mathrm{bFGF}$ and $1 \mu \mathrm{mol} / \mathrm{l}$ dexamethasone in the standard fashion $[30,31]$. These cells showed the specific characteristics of VSMC and endothelial cells by immunohistochemical examination and morphological observation. All the cells were used within passage 5 to 6 .

Measurement of HGF in conditioned medium. Human VSMC were seeded on 6-well plates at a density of $5 \cdot 10^{4} \mathrm{cells} / \mathrm{cm}^{2}$ and cultured to confluence. After replacing the medium with fresh DSF (defined serum-free medium) with or without PDGF (10 $\mathrm{ng} / \mathrm{ml}$; Biosource, Camarillo, Calif., USA) and following the culture with or without cAMP agents (forskolin or 8-bromocAMP or Dibutyl-cAMP or PDE3-Inhibitor) for $24 \mathrm{~h}$, the concentration of HGF in the medium was measured by enzyme immunoassay using anti-human HGF antibodies [28]. DSF medium was supplemented with insulin $\left(5 \cdot 10^{-7} \mathrm{~mol} / \mathrm{l}\right)$, transferrin $(5 \mathrm{mg} / \mathrm{ml})$ and ascorbate $(0.2 \mathrm{mmol} / \mathrm{l})$, as described [32].

Measurement of intracellular cAMP concentration. VSMC were grown to confluence and then exposed to PDGF with or without cAMP agents for $48 \mathrm{~h}$. Intracellular cAMP was measured using an enzyme-immunoassay kit from Amersham (Buckinghamshire, UK). In brief, culture medium was removed at the indicated times and cells were washed twice in phosphate-buffered saline and a third time in the same buffer containing 3-isobutyl-1-methylxanthine. Cells were then lysed by the addition of ice-cold trichloroacetic acid $(5 \%)$. The trichloroacetic acid-soluble supernatant was removed from the well, extracted three times with $10 \mathrm{ml}$ ether, dried, and resuspended in $0.4 \mathrm{ml}$ of sodium acetate buffer ( $\mathrm{pH}$ 6.2) per sample. The enzyme immunoassay was then carried out.

Co-culture of endothelial cells with VSMC. Endothelial cells were seeded onto cell culture inserts (Becton Dickinson, Belgium, $3.0 \mu \mathrm{mol} / 1$ pore size) and grown in $10 \%$ DMEM. Vascular smooth muscle cells were seeded onto 6-well plates, maintained in $10 \%$ DMEM and placed in DSF for $48 \mathrm{~h}$ post-confluence [33]. At $80 \%$ confluence, the inserts containing endothelial cells were put into the wells containing the quiescent confluent VSMC. Endothelial cells were co-cultured for 4 days with VSMC in fresh DSF medium containing cilostazol and the cell number was counted. An index of cell proliferation was determined using a WST-cell counting kit, since this compound produces a highly water-soluble formazan dye, which makes the assay procedure easier to perform [34]. Hypoxia was induced with an anaerobic device. Briefly, a hypoxic condition was achieved using BBL GasPak (Becton Dickson, Cockeysville, Md., USA), which catalytically reduces $\mathrm{O}_{2}$ to undetectable levels [35].

The effect of endogenously produced HGF in a co-culture of VSMC with human endothelial cells was examined by a neutralization procedure, using rabbit anti-human HGF antibody [5]. For the antibody, the IgG fraction (purified with protein A-agarose) was able to neutralize a biological activity of 10 $\mathrm{ng} / \mathrm{ml} \mathrm{HGF}$, at a concentration of $10 \mu \mathrm{g} / \mathrm{ml}$. Normal rabbit serum $\mathrm{IgG}$ fraction $(10 \mu \mathrm{g} / \mathrm{ml})$ was used as a control.

Materials. PGE1 was obtained from Ono Pharmaceutical Company (Osaka, Japan). Cilostazol was donated by Otsuka Pharmaceutical Company (Osaka, Japan).

Statistical analysis. All values are expressed as means \pm SEM. All experiments were carried out at least three times. Analysis of variance with subsequent Duncan's test was used to determine the significance of differences in multiple comparisons. Differences with $p$ values of less than $p<0.05$ were considered statistically significant.

\section{Results}

Inhibition of neointimal formation by cilostazol. Treatment with cilostazol resulted in inhibition of neointimal formation after balloon injury in non-diabetic rats at 2 weeks after balloon injury $(p<0.01$, Fig. 1). Of importance, treatment with cilostazol also resulted in a significant reduction in the ratio of neointimal to medial area even in diabetes model rats (Fig.1B). In addition, cilostazol increased the ratio of lumen to total area in non-diabetic and diabetic rats $(p<0.01$, Fig. $1 C)$. There was no significant difference in medial area among the groups.

Stimulatory effect of cilostazol on vascular HGF concentration. As HGF is one of the key molecules in endothelial function and pathogenesis of vascular injury, the concentration of endogenous vascular HGF might be important to determine the fate of endothelial cells. Vascular HGF concentration in injured vessels of non-diabetic rats was decreased as compared to that in intact vessels $(p<0.01$, Fig. 2$)$. Moreover, vascular HGF concentration in injured vessels of diabetic rats was further reduced compared with non-diabetic rats ( $p<0.05$, Fig. 2). Probably, the decrease in vascular HGF would contribute to the delay of the reendothelialization process after balloon injury. Cilostazol treatment attenuated the decrease in vascular HGF concentration in non-diabetic rats $(p<0.05$, Fig. 2). The down-regulated HGF in injured vessels of diabetic rats was also increased by cilostazol $(p<0.05$, Fig. 2$)$, accompanied with the increase in serum HGF concentration (STZ (-): Vehicle; $0.36 \pm$ 0.10 , cilostazol; $2.20 \pm 0.90 \mathrm{ng} / \mathrm{ml}, p<0.05, n=6$ ) 
A
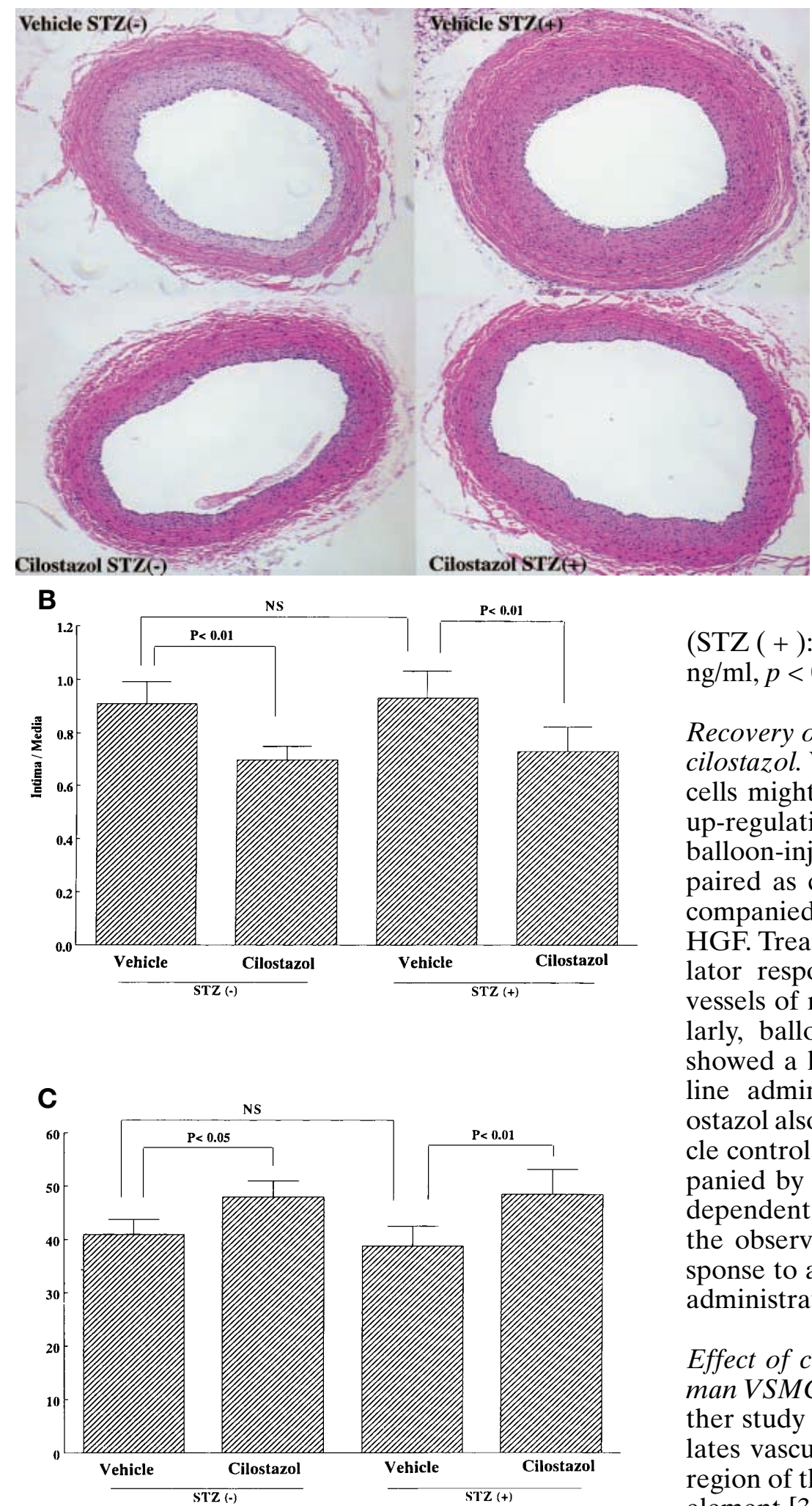

Fig. 1 (A-C). A Typical example of carotid arteries of rats treated with cilostazol or vehicle after balloon injury in non-diabetic and diabetic rats. B, C Effect of the oral administration of cilostazol on ratio of neointimal to medial area (B) and lumen area $(\mathbf{C})$ at 2 weeks after balloon injury. $n=10$ samples (sections) from 10 different animals per group. Vehicle: balloon-injured carotid artery treated with vehicle. Cilostazol: balloon-injured carotid artery treated with cilostazol, STZ (-), non-diabetic rats; STZ $(+)$, streptozotosin-induced diabetic rats

$(\mathrm{STZ}(+)$ : Vehicle; $0.30 \pm 0.18$, cilostazol; $2.97 \pm 2.64$ $\mathrm{ng} / \mathrm{ml}, p<0.05, n=6)$.

Recovery of endothelial function by administration of cilostazol. We postulated that recovery of endothelial cells might occur after cilostazol treatment through up-regulation of HGF. The vasodilator response of balloon-injured vessels in non-diabetic rats was impaired as compared to intact vessels $(p<0.01)$, accompanied by a significant decrease in vascular HGF. Treatment with cilostazol improved the vasodilator response to acetylcholine of balloon-injured vessels of non-diabetic rats $(p<0.05$, Fig. 3A). Similarly, balloon-injured vessels of diabetic rats also showed a lack of vasodilator response to acetylcholine administration (Fig.3B). Treatment with cilostazol also resulted in dilation compared to the vehicle control in diabetic rats ( $p<0.05$, Fig. 3B), accompanied by the induction of HGF. The endotheliumdependent dilation of arteries was also supported by the observation that the increase in vasodilator response to acetylcholine was completely abolished by administration of L-NAME (data not shown).

Effect of cilostazol on local HGF production in human VSMC. We used an in vitro culture system to further study the mechanisms by which cilostazol simulates vascular HGF production. Since the promoter region of the HGF gene contains a cAMP responsive element [36], we thought that cilostazol directly activates $\mathrm{HGF}$ through cAMP accumulation. Incubation with forskolin $(30 \mu \mathrm{mol} / \mathrm{l})$ as well as 8 -bromo cAMP $(1 \mathrm{mmol} / \mathrm{l})$ stimulated local HGF production in human aortic VSMC in a time-dependent manner (Fig.4A). Similarly, treatment with cilostazol, a PDE3 inhibitor, as well as prostaglandin E stimulated 


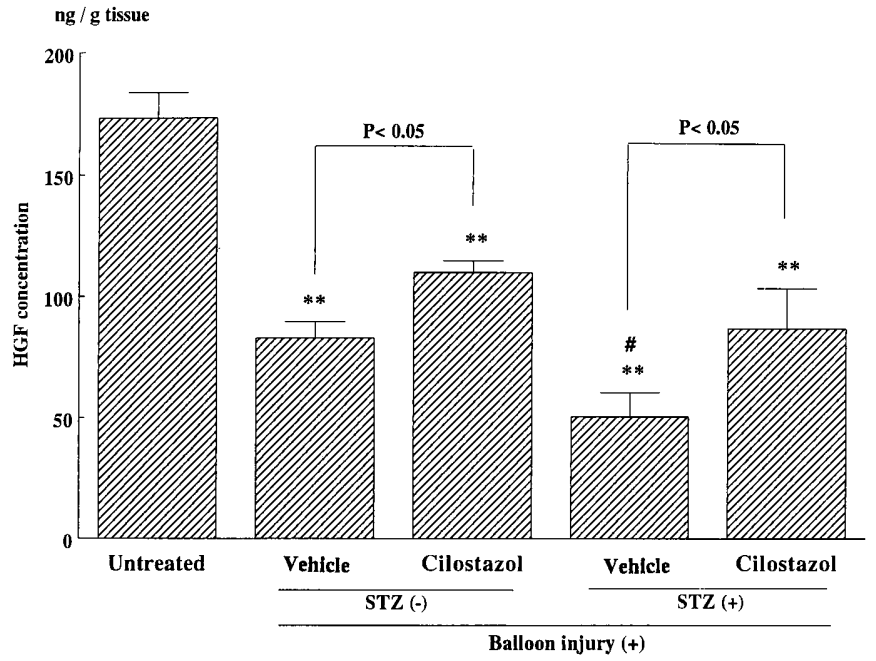

Fig. 2. Endogenous HGF concentration in injured carotid arteries. $n=8$ per group. Untreated: uninjured intact rat carotid artery treated with vehicle; Vehicle: injured rat carotid artery treated with vehicle; Cilostazol: injured rat carotid artery treated with cilostazol; STZ (-), non-diabetic rats; STZ (+ ), streptozotosin-induced diabetic rats. $* * p<0.01$ vs untreated, $\# p<0.05$ vs vehicle STZ $(-)$
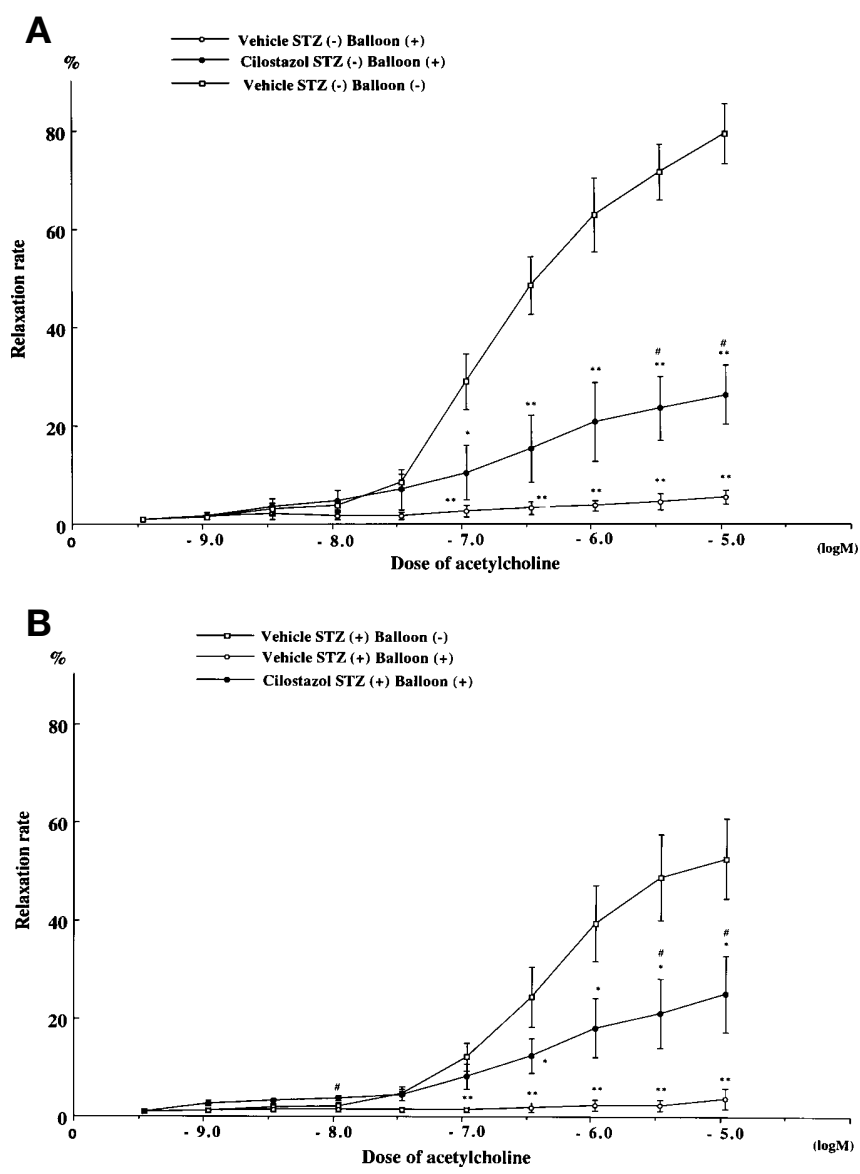

Fig.3 (A, B). Vasodilator properties in response to acetylcholine in non-diabetic rats $(\mathbf{A})$ and diabetic rats $(\mathbf{B}) . n=4$ per group. STZ (-), non-diabetic rats; STZ (+ ), streptozotosin-induced diabetic rats; Balloon (-), uninjured vessel; Balloon $(+)$, injured vessel. ** $p<0.01$ vs Balloon $(-),{ }^{*} p<0.05$ vs Balloon $(-), \# p<0.05$ vs Vehicle Balloon $(+)$

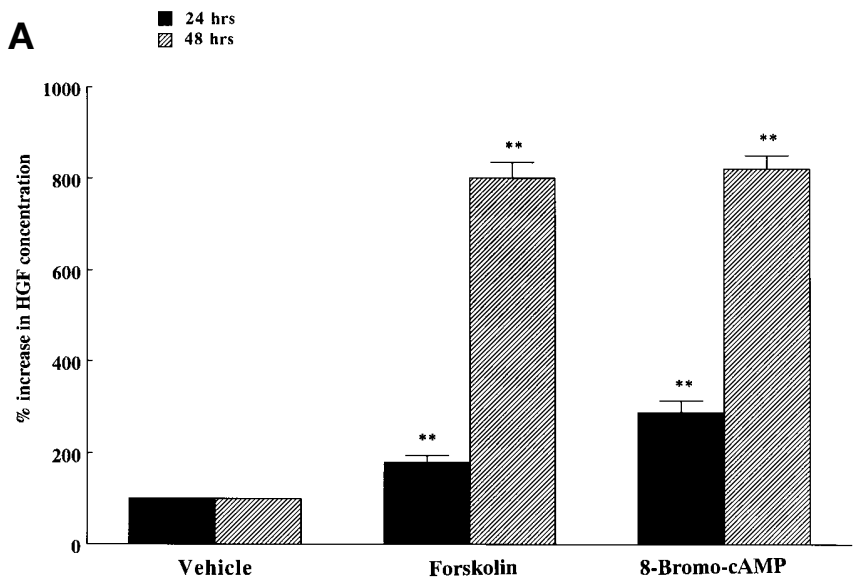

\section{B}

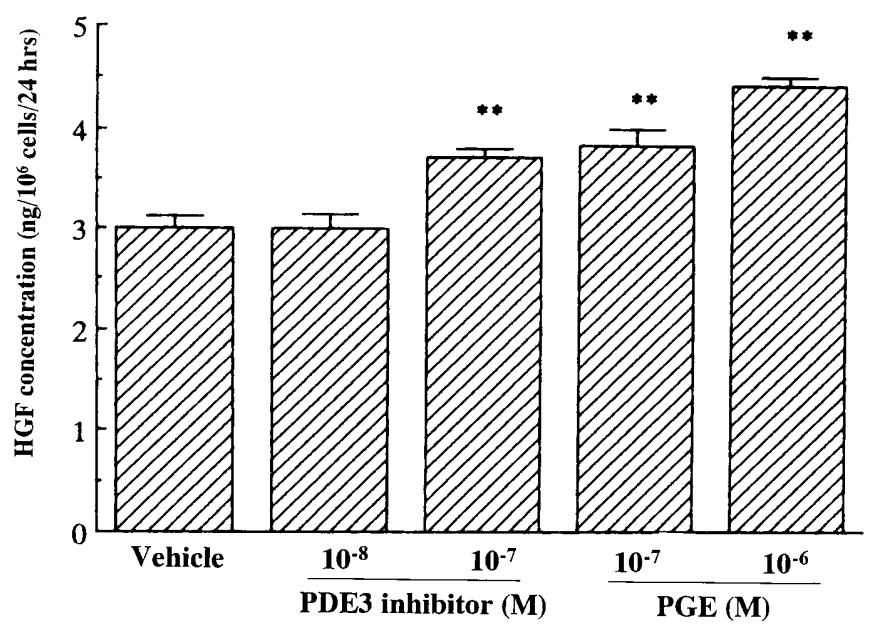

Fig. 4 (A, B). A Time-dependent stimulatory effects of forskolin and 8-bromo-cAMP on local HGF concentration in human aortic VSMC. $n=8$ per group. Vehicle: VSMC in serum-free medium treated with vehicle; Forskolin: VSMC treated with forskolin $(30 \mu \mathrm{mol} / \mathrm{l})$; 8-Bromo-cAMP: VSMC treated with 8bromo-cAMP $(1 \mathrm{mmol} / \mathrm{l}) .{ }^{* *} p<0.01$ vs vehicle. 24 hours $(\boldsymbol{\square})$; 48 hours (囚). B Dose-dependent stimulatory effects of cilostazol and PGE1 on local HGF concentration in human aortic VSMC $n=8$ per group. Vehicle: VSMC in serum-free medium treated with vehicle; PDE3 inhibitor: VSMC treated with cilostazol $\left(10^{-8}-10^{-7} \mathrm{~mol} / \mathrm{l}\right)$ : PGE: VSMC treated with $\mathrm{PGE}_{1}$ $\left(10^{-7}-10^{-6} \mathrm{~mol} / \mathrm{l}\right){ }^{* *} p<0.01 \mathrm{vs}$ vehicle. Values are expressed as HGF concentration adjusted for cell number

local HGF production in human aortic VSMC in a dose-dependent manner (Fig. 4B). cAMP accumulation induced by forskolin, 8-bromo-cAMP, dibutylcAMP and cilostazol showed a dose-dependent stimulatory effect on local HGF production $24 \mathrm{~h}$ after stimulation in human aortic VSMC (Fig. 5A). The increase in local HGF production by cAMP was still observed in VSMC treated with PDGF (Fig. 5B). The increase in local HGF production was at least in part due to the accumulation of cAMP, as (R)-p-adenosine-3',5'-cyclic phosphorothioate (an inhibitor of the cAMP-dependent protein kinase; Rp-cAMP) significantly attenuated the increase in local HGF pro- 
A

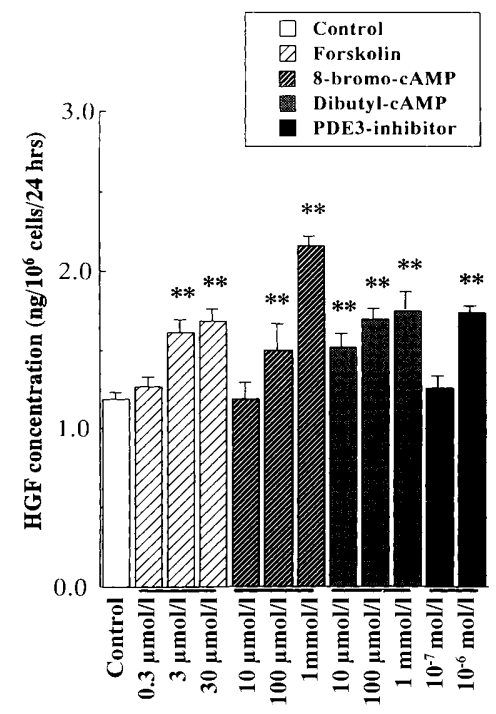

C

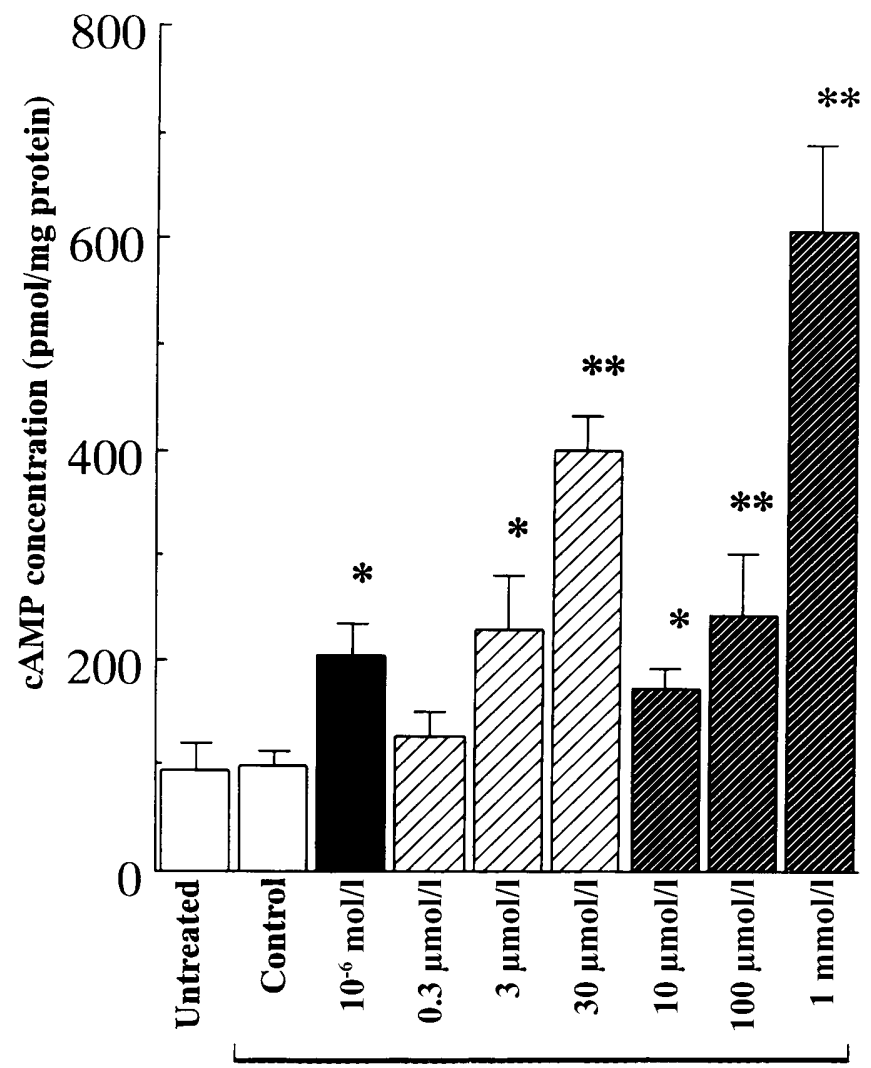

PDGF (2.5ng/ml)

B

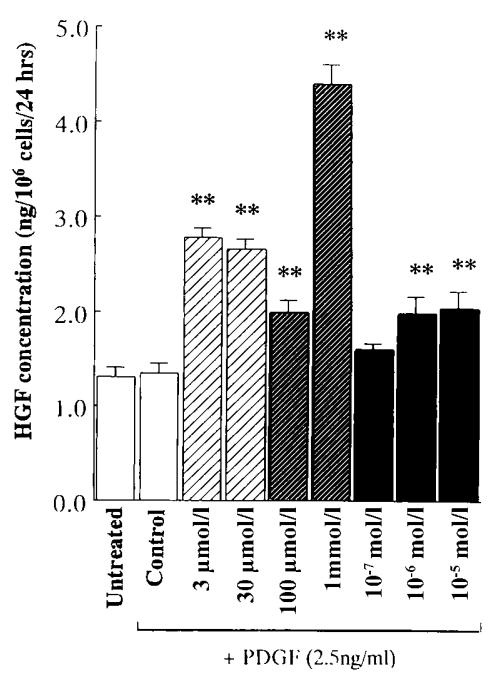

duction by 8-bromo-cAMP (8-bromo-cAMP: $5.433 \pm$ 0.149 vs 8-bromo-cAMP + Rp-cAMP: $4.485 \pm 0.143$ $\mathrm{ng} / 10^{6}$ cells $\left./ 24 \mathrm{~h}, p<0.01\right)$. Indeed, cAMP concentration was significantly increased by cilostazol as well as forskolin and 8-bromo-cAMP in a dose-dependent manner, even under PDGF stimulation in human aortic VSMC (Fig. 5C).

We used a co-culture system to examine the stimulatory effect of cilostazol on local HGF production in the growth of endothelial cells. Given the inhibitory action of endothelial cells on VSMC growth, stimulation of local HGF production by cAMP-inducing agents such as cilostazol could have therapeutic value against VSMC growth. Co-culture of VSMC with endothelial cells resulted in a significant increase in the number of endothelial cells as compared to endothelial cells alone (co-culture (-); $100 \%$, co-culture $(+) ; 118.6 \pm 2.2 \% *, * p<0.01)$, showing that factors that stimulated endothelial cells were secreted by VSMC. Incubation of cilostazol with VSMC co-cultured with endothelial cells resulted in a significant increase in the number of endothelial cells as compared to vehicle treatment (Fig. 6). In contrast, addition of neutralizing anti-HGF antibody abolished the endothelial cell growth induced by cilostazol (Fig.6). Moreover, we examined the effects of cAMP accumulation induced by cilostazol in more pathophysiological conditions such as hypoxia. We used a co-culture system of VSMC with endothelial cells under hypoxia. Hypoxic treatment of endothelial cells stimulated endothelial cell death in a co-culture of VSMC (upper well) with endothelial cells (lower well). Addition of cilostazol as well as forskolin showed an attenuation of endothelial cell death induced by hypoxia in the co-culture system. This attenuation by cilostazol was mediated by an increase 

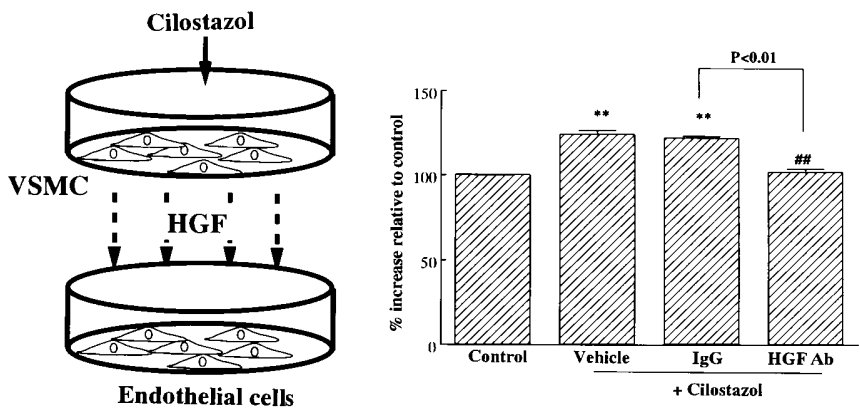

Fig. 6. Inhibition of stimulatory effect of cilostazol on growth of endothelial cells by addition of neutralizing anti-HGF antibody in a co-culture system. $n=8$ per group. Control, untreated cells; Vehicle, cells treated with vehicle; IgG, cells treated with IgG control; HGF-Ab, cells treated with anti-HGF antibody; + Cilostazol, cells treated with cilostazol $\left(10^{-6} \mathrm{~mol} / \mathrm{l}\right)$. $* * p<0.01$ vs control, $* * p<0.01$ vs vehicle. Values are expressed as percentage of control

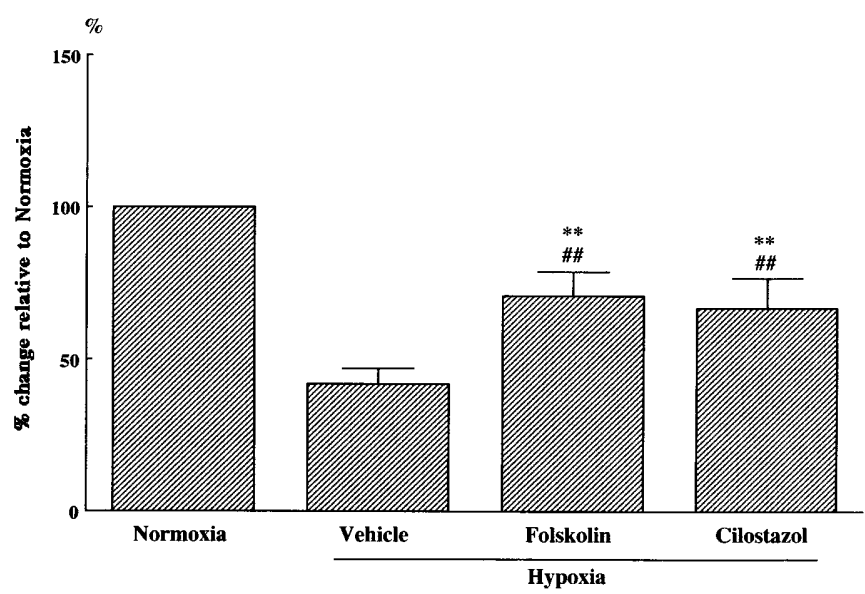

Fig. 7. Inhibition of hypoxia-induced endothelial cell death by forskolin and cilostazol in a co-culture system. $n=8$ per group. Normoxia, untreated cells under normoxic conditions; Vehicle, cells treated with vehicle under hypoxic conditions; Forskolin, cells treated with forskolin $(30 \mu \mathrm{mol} / \mathrm{l})$ under hypoxic conditions; Cilostazol, cells treated with cilostazol $\left(10^{-6} \mathrm{~mol} / \mathrm{l}\right)$ under hypoxic conditions. $* p<0.01$ vs normoxia, $\# p<0.01$ vs vehicle. Values are expressed as absorbance units.

in local HGF production, as neutralizing anti-HGF antibody diminished the attenuation by cilostazol (data not shown). Overall, these results show that release of vascular HGF stimulated by cilostazol enhanced endothelial cell growth and inhibited cell death induced by hypoxia.

\section{Discussion}

Restenosis after angioplasty still remains an issue in the field of cardiovascular disease, since the longterm effectiveness of this procedure is limited by the development of restenosis in over $40 \%$ of patients [37-39]. Several investigators have reported the po- tential effect of cilostazol to reduce restenosis in a rat balloon-injury model and in clinical studies [9-15]. The mechanism of restenosis reduction by cilostazol is considered to be mainly inhibition of SMC migration and proliferation. Because SMC migration and proliferation are induced by various growth factors released from activated platelets, cilostazol as an anti-platelet drug would suppress the secretion of such growth factors from platelets [7]. Moreover, cilostazol has a direct effect on the proliferation of SMC, as it inhibited cell cycle progression of VSMC through up-regulation of anti-oncogenes, p53-p21 [6]. Consistent with previous reports [8], we showed that cilostazol inhibited neointimal formation after balloon injury in a non-diabetic rat model. A single model however, might be far from the clinical situation. As diabetes presents in many coronary heart disease patients undergoing angioplasty, we evaluated the effect of cilostazol on neointimal formation after balloon injury in a diabetic rat model. Expectedly, treatment with cilostazol caused a significant inhibition of neointimal neointimal formation after balloon injury in a diabetic model.

This present study provides further information about the anti-proliferative action of cilostazol on neointimal formation after balloon injury. Cilostazol stimulated vascular HGF production both in non-diabetic and diabetic rats. Stimulation of vascular HGF production by cilostazol is supported by several lines of evidence. Firstly, cilostazol increased vascular HGF production in balloon-injured blood vessels in non-diabetic and diabetic rats, and stimulated local HGF production in human cultured VSMC and endothelial cells. Secondly, the promoter region of the HGF gene contains a cAMP responsive element [36]. Thirdly, the increase in cAMP concentration by forskolin as well as 8-bromo-cAMP and cilostazol was confirmed. Fourthly, the increase in HGF concentration was attenuated by Rp-cAMP (an inhibitor of the cAMP-dependent protein kinase). An increase in vascular HGF production could stimulate the process of re-endothelialization, potentially resulting in the inhibition of neointimal formation, as endothelial cells release multiple anti-proliferative vasoactive substances $\left(\mathrm{PGI}_{2}, \mathrm{NO}, \mathrm{CNP}\right)$. Of note, the vasodilator response of balloon-injured coronary arteries was restored by treatment with cilostazol as compared to vehicle. In addition, this restoration of vasodilatation was completely abolished by administration of N-nitro-L-arginine methyl ester (L-NAME), suggesting that rapid regeneration or stabilization of endothelial cells might be related to recovery of the vasodilator response to acetylcholine. Increased local HGF production by cilostazol could participate in the improvement of endothelial dysfunction observed in our study.

We demonstrated that incubation of cilostazol with VSMC co-cultured with endothelial cells result- 
ed in an increase in the number of endothelial cells as compared to vehicle, through induction of vascular HGF. Of note, even in pathophysiological conditions such as hypoxia, incubation with cilostazol attenuated hypoxia-induced endothelial cell death through increased local HGF production. Thus, increased local HGF production itself, by cilostazol, could have therapeutic value against abnormal VSMC growth through stimulation of re-endothelialization, in addition to the blockade of VSMC growth.

In this study, we did not assess to what extent reendothelialization occurred from the administration of cilostazol. However, we have previously reported that HGF has potent mitogenic actions on the regrowth of endothelial cells and that the area covered by endothelial cells, assessed by evans-blue staining, was increased in balloon injured vessels transfected with HGF gene (control; $39 \pm 9 \%$, HGF; $72 \pm 18 \%$, $p<0.01)$ [21]. As cilostazol induced the expression of HGF through the increase in cAMP in vitro and in vivo, regrowth of endothelial cells by cilostazol was considered to inhibit restenosis after balloon injury and improve endothelial function.

In addition, local HGF production in VSMC is negatively regulated by high $\mathrm{D}$-glucose through TGF- $\beta$ activation [40]. These findings are important in understanding the pathogenesis of endothelial dysfunction observed in cardiovascular disease, as restenosis after angioplasty is a common complication in diabetes. It can be hypothesized that disruption of the autocrine-paracrine local HGF system, which maintains endothelial cell growth by high glucose could result in abnormal growth of VSMC and endothelial dysfunction. Therefore, we further examined the pathophysiological roles of the vascular HGF system in diabetes. Expectedly, the vasodilator response of blood vessels was markedly diminished in diabetes, accompanied by a decrease in vascular HGF concentration. In contrast, administration of cilostazol increased local HGF expression, associated with the inhibition of neointimal formation and improvement of endothelial dysfunction in a diabetes model.

The effects of high glucose condition on neointimal formation after balloon injury has been reported to be different between human and rats, although diabetes is well known to increase restenosis in humans. A reduced neointimal formation in diabetic rats has already been observed [41]. It has also been reported that high glucose accelerates proliferation of rat vascular smooth muscle cells through PKC pathway [42, 43] or up-regulation of TGF- $\beta$ [40] and that the early proliferative response of smooth muscle cells in re-injured aortae was increased in diabetic rats [44]. In our study, the ratio of neointima to media (I:M) after balloon injury was not different between diabetic and non-diabetic rats.

Overall, we showed that cilostazol has dual inhibitory actions against neointimal formation, inhibition of VSMC growth and stimulation of local HGF production, resulting in the rapid regeneration of endothelial cells.

Acknowledgements. We wish to thank M. Yamashita, R. Kozai and M. Tamakoshi for their excellent technical assistance. This work was partially supported by grants from the Japan Health Sciences Foundation and the Japan Cardiovascular Research Foundation, a Japan Heart Foundation Research Grant, a Grant-in-Aid from The Ministry of Public Health and a Grant-in-Aid from The Ministry of Education, Science, Sports and Culture.

\section{References}

1. Califf RM, Fortin DF, Frid DJ et al. (1991) Restenosis after coronary angioplasty: an overview. J Am Coll Cardiol 17: 2B-13

2. Mintz GS, Popma JJ, Pichard AD et al. (1996) Arterial remodeling after coronary angioplasty: a serial intravascular ultrasound study. Circulation 94: 35-43

3. Liu MW, Roubin GS, King SB 3rd (1989) Restenosis after coronary angioplasty. Potential biologic determinants and role of intimal hyperplasia. Circulation 79: 1374-1387

4. McBride W, Lange RA, Hillis LD (1988) Restenosis after successful coronary angioplasty. Pathophysiology and prevention. N Engl J Med 30: 1734-1737

5. Morishita R, Higaki J, Hayashi S et al. (1997) Role of hepatocyte growth factor in endothelial regulation: Prevention of high D-glucose-induced endothelial cell death by prostaglandins and phosphodiesterase type 3 inhibitor. Diabetologia 40: 1053-1061

6. Hayashi S, Morishita R, Matsushita H et al. (2000) Cyclic AMP inhibited proliferation of human aortic vascular smooth muscle cells, accompanied by induction of p53 and p21. Hypertension 35: 237-243

7. Takahashi S, Oida K, Fujiwara R et al. (1992) Effect of cilostazol, a cyclic AMP phosphodiesterase inhibitor, on the proliferation of rat aortic smooth muscle cells in culture. J Cardiovasc Pharmacol 20: 900-906

8. Ishizaka N, Taguchi J, Kimura Y et al. (1999) Effects of a single local administration of cilostazol on neointimal formation in balloon-injured rat carotid artery. Atherosclerosis 142: 41-46

9. Tsuchikane E, Fukuhara A, Kobayashi T et al. (1999) Impact of cilostazol on restenosis after percutaneous coronary balloon angioplasty. Circulation 6: 21-26

10. Park S, Lee CW, Kim H et al. (2000) Effects of cilostazol on angiographic restenosis after coronary stent placement. Am J Cardiol 1: 499-503

11. Yamasaki M, Hara K, Ikari Y et al. (1998) Effects of cilostazol on late lumen loss after Palmaz-Schatz stent implantation. Cathet Cardiovasc Diagn 44: 387-391

12. Sekiya M, Funada J, Watanabe K, Miyagawa M, Akutsu H (1998) Effects of probucol and cilostazol alone and in combination on frequency of poststenting restenosis. Am J Cardiol 15: 144-147

13. Kunishima T, Musha H, Eto F et al. (1997) A randomized trial of aspirin versus cilostazol therapy after successful coronary stent implantation. Clin Ther 19: 1058-1066

14. Take S, Matsutani M, Ueda H et al. (1997) Effect of cilostazol in preventing restenosis after percutaneous transluminal coronary angioplasty. Am J Cardiol 15: 1097-1099

15. Tsutsui M, Shimokawa H, Higuchi S et al. (1996) Effect of cilostazol, a novel anti-platelet drug, on restenosis after 
percutaneous transluminal coronary angioplasty. Jpn Circ J 60: 207-215

16. Dzau VJ (1993) The role of mechanical and humoral factors in growth regulation of vascular smooth muscle cells and cardiac myocytes. Curr Opin Nephrol Hypertens 2: 27-32

17. Gibbons GH, Dzau VJ (1994) The emerging concept of vascular remodeling. N Engl J Med 30: 1431-1438

18. Laitinen M, Hartikainen J, Hiltunen MO et al. (2000) Catheter-mediated vascular endothelial growth factor gene transfer to human coronary arteries after angioplasty. Hum Gene Ther 20: 263-270

19. Dulak J, Jozkowicz A, Guevara I, Dembinska-Kiec A (1999) Gene transfer of vascular endothelial growth factor and endothelial nitric oxide synthase implications for gene therapy in cardiovascular diseases. Pol J Pharmacol 51: 233-241

20. Asahara T, Bauters C, Pastore C et al. (1995) Local delivery of vascular endothelial growth factor accelerates reendothelialization and attenuates intimal hyperplasia in balloon-injured rat carotid artery. Circulation 1 : 2793-2801

21. Hayashi K, Nakamura S, Morishita R et al. 2000 In vivo transfer of human hepatocyte growth factor gene accelerates re-endothelialization and inhibits neointimal formation after balloon injury in rat model. Gene Ther 7: 1664-1671

22. Tesfamariam B, Brown ML, Cohen RA (1991) Elevated glucose impairs endothelium-dependent relaxation by activating protein kinase C. J Clin Invest 87: 1643-1648

23. Hsueh WA, Anderson PW (1992) Hypertension, the endothelial cell, and the vascular complications of diabetes mellitus. Hypertension 20: 253-263

24. Kim EM, Grace MK, Welch CC, Billington CJ, Levine AS (1999) STZ-induced diabetes decreases and insulin normalizes POMC mRNA in arcuate nucleus and pituitary in rats. Am J Physiol 276: R1320-R1326

25. Morishita R, Gibbons GH, Ellison KE et al. (1993) Single intraluminal delivery of antisense cdc 2 kinase and PCNA oligonucleotides results in chronic inhibition of neointimal hyperplasia. Proc Natl Acad Sci USA 90: 8474-8478

26. Morishita R, Gibbons GH, Ellison KE et al. (1994) Intimal hyperplasia after vascular injury is inhibited by antisense cdk 2 kinase oligonucleotides. J Clin Invest 93: 1458-1464

27. Nakamura Y, Morishita R, Higaki J, Kida I, Aoki M, Moriguchi A et al. (1995) Expression of local hepatocyte growth factor system in vascular tissues. Biochem Biophys Res Commun 215: 483-488

28. Yamada A, Matsumoto K, Iwanari H et al. (1995) Rapid and sensitive enzyme-linked immunosorbent assay for measurement of HGF in rat and human tissues. Biomed Res 16: 105-114

29. Nakajima M, Toda N (1986) Prejunctional and postjunctional actions of prostaglandins F2 alpha and I2 and carbocyclic thromboxane A2 in isolated dog mesenteric arteries. Eur J Pharmacol 120: 309-318
30. Wertheimer SJ, Myers CL, Wallace RW, Parks TP (1992) Intercellular adhesion molecule-1 gene expression in human endothelial cells. J Biol Chem 267: 12030-12035

31. Bonin PD, Leadley RJ, Erickson LA (1993) Growth factorinduced modulation of endothelial-1 binding to human smooth muscle cells. J Cardiovasc Pharmacol 22: S125-S127

32. Libby P, O $\mu$ Brien KV (1983) Culture of quiescent arterial smooth muscle cells in a defined serum-free medium. J Cell Physiol 115: 217-223

33. Morishita R, Gibbons GH, Pratt RE et al. (1994) Autocrine and paracrine effects of atrial natriuretic peptide gene transfer on vascular smooth muscle and endothelial cellular growth. J Clin Invest 94: 824-829

34. Ishiyama M, Shiga M, Sasamoto K, Mizoguchi M, He P (1993) A new sulfonated tetrazolium salt that produces a highly water-soluble formazan dye. Chem Pharm Bull (Tokyo) 41: 1118-1122

35. Shweiki D, Itin A, Soffer D, Keshet E (1992) Vascular endothelial growth factor induced by hypoxia may mediate hypoxia-initiated angiogenesis. Nature 359: 843-845

36. Nakano N, Morishita R, Moriguchi A et al. (1998) Negative regulation of local hepatocyte growth factor (HGF) expression by angiotensin II and transforming growth factor$\beta$ in blood vessels: potential role of HGF in cardiovascular disease. Hypertension 32: 444-451

37. Libby P, Schwartz D, Brogi E, Tanaka H, Clinton SK (1992) A cascade model for restenosis: A special case of atherosclerosis progression. Circulation 86: III47-III52

38. Casscells W (1992) Migration of smooth muscle and endothelial cells: Critical events in restenosis. Circulation 86: 723-729

39. Clowes AW, Clowes MM, Fingerle J, Reidy MA (1989) Regulation of smooth muscle cell growth in injured artery. J Cardiovasc Pharmacol 14: S12-S15

40. Morishita R, Nakamura S, Nakamura Yet al. (1997) Potential role of an endothelium-specific growth factor, hepatocyte growth factor, on endothelial damage in diabetes. Diabetes 46: 138-142

41. Edelman ER, Pukac LA, Karnovsky MJ (1993) Protamine and protamine-insulins exacerbate the vascular response to injury. J Clin Invest 91: 2308-2313

42. Yamamoto M, Acevedo-Duncan M, Chalfant CE, Patel NA, Watson JE, Cooper DR (2000) Acute glucose-induced downregulation of PKC-betaII accelerates cultured VSMC proliferation. Am J Physiol Cell Physiol 279: C587-C595

43. Hall JL, Matter CM, Wang X, Gibbons GH (2000) Hyperglycemia inhibits vascular smooth muscle cell apoptosis through a protein kinase C-dependent pathway. Circ Res 87: $574-580$

44. Winocour PD, Hryhorenko L (1995) Spontaneous diabetes in BB Wistar rats causes small increases in the early proliferative response of smooth muscle cells in re-injured aortae. Exp Mol Pathol 63: 161-174 